\section{Botulism Outbreak from Drinking Prison-Made Illicit Alcohol in a Federal Correctional Facility - Mississippi, June 2016}

Lindsey McCrickard, DVM ${ }^{1,2}$; Mariel Marlow, $\mathrm{PhD}^{1,2}$; Julie L. Self, $\mathrm{PhD}^{1,2}$; Louise Francois-Watkins, $\mathrm{MD}^{2}$; Kevin Chatham-Stephens, $\mathrm{MD}^{2}$; Jannifer Anderson ${ }^{3}$; Sheryl Hand ${ }^{3}$; Kathryn Taylor, MD ${ }^{3}$; Jennifer $\mathrm{Hanson}^{3}$; Keiundria Patrick ${ }^{3}$; Carolina Luquez, $\mathrm{PhD}^{2}$; Janet Dykes, $\mathrm{MS}^{2}$; Suzanne R. Kalb, $\mathrm{PhD}^{4}$; Kaitlin Hoyt ${ }^{4}$; John R. Barr, PhD ${ }^{4}$; Todd Crawford, $\mathrm{MSN}^{5}$; Anthony Chambers, $\mathrm{MD}^{5}$; Brian Douthit ${ }^{5}$; Robert Cox, $\mathrm{MD}^{6}$; Matt Craig, $\mathrm{MD}^{6}$; John Spurzem, $\mathrm{MD}^{6}$; Joseph Doherty, $\mathrm{MD}^{6}$; Michael Allswede, $\mathrm{DO}^{7}$; Paul Byers, $\mathrm{MD}^{3}$; Thomas Dobbs, $\mathrm{MD}^{3}$

On June 9, 2016, the Mississippi Poison Control Center and the Mississippi State Department of Health (MSDH) notified CDC of five suspected cases of botulism, a potentially fatal neuroparalytic illness $(1)$, in inmates at a medium-security federal correctional institution (prison A). By June 10, a total of 13 inmates were hospitalized, including 12 in Mississippi and one in Oklahoma (the inmate in Oklahoma had been transferred there after his exposure for reasons unrelated to his illness). MSDH, Oklahoma State Department of Health, Bureau of Prisons, and $\mathrm{CDC}$ conducted an investigation to identify the source and scope of the outbreak, and to develop recommendations.

Prison A staff members suspected that an alcoholic beverage, illicitly made by inmates and known as "hooch" or "pruno," was the source of the outbreak. Among 33 inmates who reported consuming hooch during June 1-19, 2016, a total of 31 (94\%) had signs or symptoms suggesting botulism. The median interval from first exposure to symptom onset was 3 days (range $=0-11$ days) (Figure). Cases were categorized using modified Council of State and Territorial Epidemiologists definitions. A confirmed case was defined as an illness in an inmate consistent with botulism that began on or after June 1, with botulinum toxin type A detected in a serum or stool specimen or Clostridium botulinum cultured from a stool specimen; a probable case was defined as an illness in an inmate with signs or symptoms of any cranial nerve palsy and extremity weakness that began on or after June 1; and a suspected case was an illness in an inmate with signs or symptoms of any cranial nerve palsy without extremity weakness that began on or after June 1.

Thirty-one cases were identified, all in men, including 19 confirmed cases (18 in Mississippi and one in Oklahoma), 10 probable cases (nine in Mississippi and one in Texas), and two suspected cases in Mississippi. Patients from Texas and Oklahoma were transferred from prison A to other prisons before their illness began as part of routine inmate transfers.
FIGURE. Botulism cases $(n=31$ ) in a federal correctional facility, by reported date of hooch exposure* and symptom onset - Mississippi, June 1-19, 2016

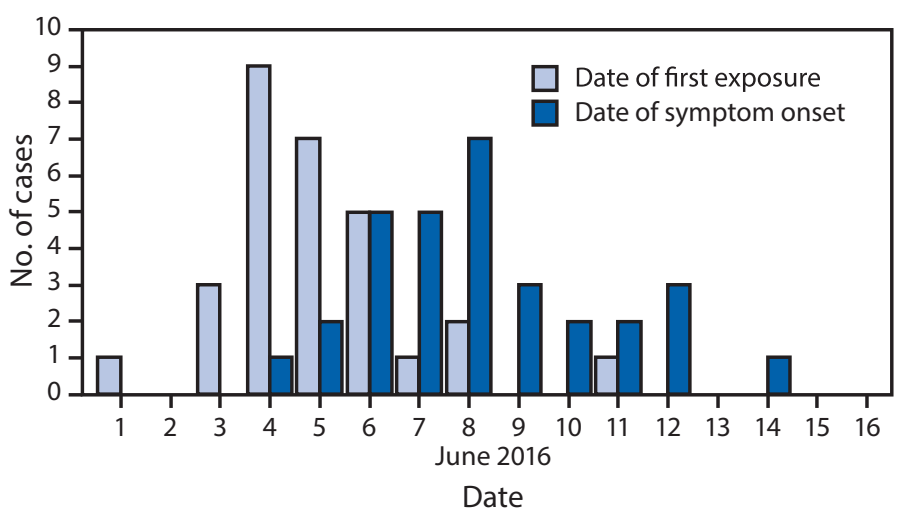

* Hooch is defined as an illicitly made alcoholic beverage. First exposure is defined as the first exposure to hooch occurring during June 1-19, 2016. Date of exposure was unknown for two inmates.

Twenty-four (77\%) patients were non-Hispanic black, six $(19 \%)$ were Hispanic white, and one identified as other non-Hispanic (3\%). The median age was 36 years (range $=23-47$ years). By the end of the outbreak, 24 inmates were hospitalized, including $15(63 \%)$ who were admitted to an intensive care unit and nine (38\%) who required intubation and mechanical ventilation; none died. Twenty $(83 \%)$ patients received botulinum antitoxin; 11 patients with mild illness did not receive antitoxin.

Medical chart abstractions (30) and interviews (30) were conducted for patients in Mississippi (29), Oklahoma (1) and Texas (1) to obtain information on hooch exposure, clinical signs and symptoms, medical management, and patients' understanding of botulism. One patient in Oklahoma was not able to be interviewed, and one patient in Texas did not have a medical chart for abstraction. Among 30 patients interviewed, 27 (90\%) had never heard of botulism, and 23 $(77 \%)$ did not know that drinking hooch could make them sick. Eleven (42\%) of 26 patients who responded to questions regarding the frequency of hooch consumption reported drinking hooch at least once a month. Some hooch exposure dates associated with this outbreak coincided with a farewell party for one inmate and the National Basketball Association finals. Although prison A staff members confiscated $>20$ gallons of hooch during the investigation, the number of circulating batches of hooch immediately before the outbreak, and the ingredients and preparation method of the batch responsible for the outbreak, are unknown. One patient reported that 
honey, potatoes, apples, and tomato paste from a bulging can were combined, hidden, and fermented in a sealed plastic bag at room temperature for 3-5 days. Possible sources of Clostridium botulinum or toxin include tomato paste, potatoes, other ingredients, or contamination from the environment. Potatoes have been hypothesized as the source of other prunoassociated outbreaks (2).

This botulism outbreak, the largest in the United States since 1978 (3), highlights the clinical spectrum of illness, ranging from total paralysis requiring intensive care and mechanical ventilation to cranial nerve complaints not requiring hospitalization (4). Facility staff members should consider the potential for increased hooch consumption during celebratory events. Educating correctional facility staff members and inmates about the risks of consuming hooch and good communication channels between facility staff members and inmates can help to identify and treat persons with botulism quickly and prevent deaths.

\section{Acknowledgments}

University of Mississippi Medical Center, Jackson, Mississippi; Baptist Medical Center, Jackson, Mississippi; Merit Health Central Medical Center, Jackson, Mississippi; Integris Southwest Medical Center, Oklahoma City, Oklahoma; Mississippi State Department of
Health; Oklahoma State Department of Health; City of Beaumont (Texas) Public Health Department; Texas Department of State Health Services; Federal Bureau of Prisons; Division of Foodborne, Waterborne, and Environmental Diseases, National Center for Emerging and Zoonotic Infectious Diseases, CDC; Strategic National Stockpile, Office of Public Health Preparedness and Response, CDC; Office of Regulatory Affairs, CDC.

${ }^{1}$ Epidemic Intelligence Service, CDC; 2 Division of Foodborne, Waterborne, and Environmental Diseases, National Center for Emerging and Zoonotic Infectious Diseases, CDC; ${ }^{3}$ Mississippi State Department of Health; ${ }^{4}$ Division of Laboratory Sciences, National Center for Environmental Health, CDC; ${ }^{5}$ Federal Bureau of Prisons, Washington, D.C.; ${ }^{6}$ University of Mississippi Medical Center, Jackson, Mississippi; ${ }^{7}$ Integris Southwest Medical Center, Oklahoma City, Oklahoma.

Corresponding Author: Lindsey McCrickard, Vjf4@cdc.gov 404-718-1607.

\section{References}

1. Sobel J. Botulism. Clin Infect Dis 2005;41:1167-73. http://dx.doi. org/10.1086/444507

2. CDC. Botulism from drinking prison-made illicit alcohol—Utah 2011. MMWR Morb Mortal Wkly Rep 2012;61:782-4.

3. CDC. US Public Health Service: botulism—New Mexico. MMWR Morb Mortal Wkly Rep 1978;27:138.

4. McCarty CL, Angelo K, Beer KD, et al. Notes from the field: large outbreak of botulism associated with a church potluck meal—Ohio, 2015. MMWR Morb Mortal Wkly Rep 2015;64:802-3. http://dx.doi. org/10.15585/mmwr.mm6429a6 